\title{
Fenologia reprodutiva e qualidade das sementes de Cabralea canjerana (Vell.) Mart
}

\author{
Reproductive phenology and seed quality of Cabralea canjerana (Vell.) Mart
}

\author{
Marciele Felippi ${ }^{\mathrm{I}}$ Maristela Machado Araújo ${ }^{\mathrm{II}}$ \\ Solon Jonas Longhi" Alessandro DalCol Lucio ${ }^{\text {III }}$
}

\section{RESUMO}

A madeira de Cabralea canjerana (Vell.) Mart. (canjerana) é de elevada qualidade, sendo indicada para confecção de móveis e construção civil, além de possuir importância paisagística. $O$ objetivo no presente estudo foi caracterizar a fenologia reprodutiva de canjerana, além de verificar os aspectos físicos e fisiológicos das sementes de diferentes árvores matrizes localizadas em remanescentes, no Municipio de Frederico Westphalen, RS. Durante três anos foram realizadas observações fenológicas mensais, em 20 indivíduos arbóreos, e após a coleta dos frutos maduros, as sementes foram analisadas no Laboratório de Silvicultura da Universidade Federal de Santa Maria, RS, comparando-se matrizes por meio do peso de mil sementes, teor de água, porcentagem e velocidade de germinação e emergência. A floração de C. canjerana é anual, persistindo de agosto a dezembro, sendo possivel encontrar botões florais, concomitantemente com frutos em desenvolvimento e maduros. Os frutos iniciam a dispersão no mês de setembro, podendo ser coletados em amplo período (setembro a março) e frutos aparentemente maduros, em diferentes árvores matrizes, não garantem elevado poder germinativo às sementes de $\boldsymbol{C}$. canjerana.

Palavras-chave: Canjerana, frutificação, germinação.

\section{ABSTRACT}

The wood of Cabralea canjerana (Vell.) Mart. (canjerana) has high quality and is suitable for making furniture and civil construction, besides having importance in landscaping. The aim of this study was to characterize the reproductive phenology of canjerana, and check the physical and physiological aspects of seeds of different matrix trees located in a remnant in the city of Frederico Westphalen-RS. For three years monthly phenological observations were carried out on 20 individuals trees and after collecting the ripe fruits, seeds were analyzed in the
Sylviculture Laboratory of the Universida Federal de Santa Maria$R S$, comparing matrixes through the weight of a thousand seeds, water content, percentage and germination and emergence speed. The flowering $\boldsymbol{C}$. canjerana is annual, persisting from August to December, being possible to find flower buds, concurrently with developing and ripe fruits. The fruits begin to spread in September being possible to be collected during a large period (September to March) and apparently ripe fruit in different matrix trees, do not guarantee high germination power to the seeds of $\boldsymbol{C}$. canjerana.

Key words: Canjerana, fruiting, germination.

\section{INTRODUÇÃO}

A sustentabilidade ecológica dos planos de manejo florestal, visando o uso múltiplo das florestas, deve incluir critérios e indicadores que garantam a manutenção da variabilidade genética das espécies e processos correlacionados. No entanto, conhecimentos básicos sobre as características que regulam os processos reprodutivos de espécies arbóreas tropicais ainda são insuficientes (KANASHIRO et al., 2002).

A fenologia estuda a ocorrência de eventos biológicos repetitivos em relação aos efeitos desencadeados pelos fatores bióticos e abióticos (LEIGHT, 1999), de forma que os estudos fenológicos possibilitam conhecer a distribuição temporal dos recursos (flores e frutos), entender a dinâmica de reprodução e regeneração das plantas, bem como a relação entre as plantas e os animais (CALVIN \&

\footnotetext{
${ }^{\mathrm{I}}$ Cordenação de Ciências Biológicas (COBIO), Universidade Tecnológica Federal do Paraná (UTFPR), 85660-000, Dois Vizinhos, PR, Brasil. E-mail: marcielefelippi@utfpr.edu.br. Autor para correspondência.

IIDepartamento de Ciências Florestais, Centro de Ciências Rurais (CCR), Universidade Federal de Santa Maria (UFSM), Santa Maria, RS, Brasil.

IIIDepartamento de Fitotecnia, CCR, UFSM, Santa Maria, RS, Brasil. 
PIÑA-RODRIGUES, 2005), assim como compreender processos de adaptabilidade e desenvolvimento de espécies em um determinado local.

Associada às fases reprodutivas e de crescimento das espécies, informações sobre as características físicas e fisiológicas de sementes são importantes para formação de lotes de qualidade para produção de mudas, uma vez que as sementes e mudas atualmente disponíveis no mercado necessitam de informações sobre procedência e número de matrizes que compõem o lote (PIÑARODRIGUES et al., 2007). A alta produção informal, muitas vezes, sem informações quanto à origem das sementes, possivelmente, está associada ao aumento da demanda por espécies nativas.

Apesar da literatura enfatizar a importância da coleta adequada de sementes, obtida em número representativo de árvores matrizes, com baixo grau de parentesco e em áreas de ocorrência natural (PIÑARODRIGUES et al., 2007), o alto custo em função da fragmentação representa um obstáculo para a coleta (SILVA \& HIGA, 2006).

A família Meliaceae está representada na região Sul do Brasil por 16 espécies, agrupadas em quatro gêneros (KLEIN, 1984). Dentre as espécies, encontra-se a Cabralea canjerana (Vellozo) Martius, conhecida como canjerana, podendo ser empregada na construção civil, recuperação de áreas degradadas, medicina popular e indústria madeireira (CARVALHO, 2003). Apesar de ser considerada uma das árvores mais valiosas no Sul do Brasil pela qualidade da madeira (REITZ et al., 1988), várias são as lacunas a serem preenchidas a seu respeito. Nesse sentido, destaca-se a definição da época adequada e do número de matrizes para coleta de sementes, como também a quantidade de sementes necessárias para a formação de lotes com elevada qualidade.

Portanto, neste trabalho, o objetivo foi caracterizar a fenologia reprodutiva de canjerana, além de verificar se sementes provenientes de diferentes árvores, coletadas na mesma região e período, apresentam semelhante potencial germinativo.

\section{MATERIAL E MÉTODOS}

O trabalho foi realizado durante o período de março de 2007 a março de 2010, cujas observações fenológicas e coleta dos frutos maduros foram realizadas no município de Frederico WestphalenRS, enquanto a análise de sementes foi efetuada no Laboratório de Silvicultura da Universidade Federal de Santa Maria, em Santa Maria-RS, e o estudo da emergência na mesma instituição, no campus de Frederico Westphalen.
A região de coleta situa-se a $566 \mathrm{~m}$ de altitude, com classificação climática, segundo Köppen, do tipo Cfa, precipitação pluvial média anual entre 1.800 e $2.100 \mathrm{~mm}$ e temperatura média anual em torno de $18^{\circ} \mathrm{C}$ (RIO GRANDE DO SUL, 2001).

$\mathrm{Na}$ área de estudo, foram observadas 20 árvores matrizes, considerando, para seleção, os indivíduos adultos, com copa bem distribuída e fuste reto, distantes pelo menos $100 \mathrm{~m}$ entre si, conforme sugerido por PINÃ-RODRIGUES et al. (2007). A distância mínima entre matrizes foi mantida, levando em consideração, o sistema sexual das plantas, que para a canjerana, pode ser hermafrodita ou dióica, de acordo com PENNINGTON (1981).

As árvores matrizes foram monitoradas mensalmente, com o auxílio de um binóculo, para observação da presença de cada fenofase, sendo a floração classificada conforme MORELLATO et al. (1989), como o período em que as árvores estavam com flores em antese e a frutificação quando os frutos encontravam-se maduros.

No início dos eventos fenológicos intensificaram-se as observações, passando para quinzenais, com a finalidade de analisar os padrões de frequência de indivíduos e ocorrência de cada fenofase, conforme indicado por NEWSTROM et al. (1994). Essas informações foram correlacionadas às condições climáticas locais, estas obtidas junto à Estação Meteorológica do Centro de Educação Superior Norte do Estado do Rio Grande do Sul (CESNORS).

$\mathrm{O}$ índice de atividade foi calculado como forma de estimar a sincronia, uma vez que indica a proporção de indivíduos amostrados em determinado evento fenológico.

Após a mudança de coloração de verde para vermelho, foram realizadas coletas diretamente nas árvores por meio de escalada e uso de podão em sete indivíduos que possuíam frutos em semelhante estádio de maturação, em meados do mês de novembro de 2007. Em seguida, os frutos de cada matriz foram armazenados em sacos plásticos e mantidos em condições de laboratório durante o período de sete dias, onde tiveram sua abertura completa, método este descrito por INOUE (1978) e, na sequência as sementes foram beneficiadas e analisadas separadamente por matriz.

A avaliação foi realizada em cada matriz para determinar o peso de mil sementes e o teor de água, conforme as Regras para Análises de Sementes (BRASIL, 2009). No teste de germinação foram utilizadas caixas de acrílico com tampa $(11 \times 11 \times 3,5 \mathrm{~cm})$ do tipo gerbox, com oito repetições de 25 sementes colocadas entre vermiculita média 
esterilizada em autoclave durante o período de $1 \mathrm{~h}$ a $120^{\circ} \mathrm{C}$, a qual foi umedecida a $60 \%$ de capacidade de retenção de água. As amostras foram colocadas em germinador Mangelsdorf, regulado à temperatura de $25 \pm 3^{\circ} \mathrm{C}$, com luz branca contínua, considerando-se, para análise, o critério botânico de emissão de $2 \mathrm{~mm}$ de raiz primária (GUI-FERREIRA \& BORGHETTI, 2004), durante 28 dias.

O teste de emergência foi realizado em casa de vegetação, utilizando-se 54 tubetes $\left(175 \mathrm{~cm}^{3}\right)$ preenchidos com substrato MecPlant Florestal $3^{\circledR} \mathrm{em}$ quatro repetições, totalizando 216 mudas para cada matriz, sendo a semeadura realizada na profundidade de aproximadamente um centímetro, com regas duas vezes ao dia e avaliação diária durante 80 dias. A emergência foi considerada a partir do aparecimento do protófilo, conforme descrito por GUI-FERREIRA \& BORGHETTI (2004).

Os dados de laboratório e viveiro foram analisados quanto à porcentagem de germinação $(\% \mathrm{G})$, emergência $(\% \mathrm{E})$, índice de velocidade de germinação (IVG) e de emergência (IVE), conforme Maguire (1962).

Os resultados foram submetidos à análise de pressuposições básicas de normalidade e homocedasticidade (SANTANA \& RANAL, 2004), utilizando os testes de Shapiro-Wilk e Bartlett, respectivamente. Quando essas pressuposições não foram atendidas, procedeu-se à transformação dos dados por meio de arc sen, sendo x o percentual de germinação e emergência. Para os casos em que as pressuposições não foram atendidas, mesmo transformando os dados, foi utilizado o teste não paramétrico de Kruskal-Wallis, recomendado por SANTANA \& RANAL (2004) e, logo após, foi aplicado o teste de Dunn, para comparações múltiplas (ZAR, 2009); todos os testes foram realizados com o auxílio do software Statistical Analysis System (SAS) 8.2.

\section{RESULTADOS E DISCUSSÃO}

A floração de $\boldsymbol{C}$. canjerana ocorreu de agosto a dezembro nos três anos de avaliação, sendo intensa no período de maior precipitação pluviométrica (setembro e outubro), de forma que, nesse contexto pode ser considerada anual, pela classificação de NEWSTRON et al. (1994) e sincrônica, conforme BENCKE \& MORELLATO (2002).

Não houve sincronia das fases reprodutivas da canjerana, porque observaram-se diversas fases fenológicas (diferenciação, início do botão floral, flores em antese, frutos em desenvolvimento e maduros) ocorrendo simultaneamente. De acordo com PENNINGTON (1981), uma das características da subespécie canjerana é ter flores e frutos maduros na mesma época, confirmando os resultados obtidos neste estudo.

A floração na transição da estação seca para úmida ou no início da estação úmida, nas florestas semideciduais, tem sido atribuída, principalmente, à redução do estresse hídrico causado pelas primeiras chuvas após o período de seca, ao aumento do fotoperíodo e à elevação da temperatura (MORELLATO, 1995).

O período de floração para a região de estudo (agosto a dezembro) assemelha-se ao descrito por MOSCHETA et al. (2002), no entanto, antecede a época citada por CARVALHO (2003) (fevereiro a março), enquanto BACKES \& IRGANG (2002) destacaram a ocorrência predominante de flores entre agosto a março. Portanto, a época de atividade da fenofase, neste trabalho, expressa variações em relação aos dados relatados, sendo, assim, complementares para a espécie.

As variações fenotípicas ou até mesmo a irregularidade produtiva entre anos consecutivos pode estar associada a fatores genéticos e ambientais, como precipitação e temperatura (FELIPPI et al., 2012), podendo expressar modificações na morfologia, anatomia, taxa fotossintética, entre outros fatores.

Os frutos maduros foram observados de setembro a março, com maior intensidade nos meses de novembro e dezembro. Assim, a frutificação pode ser considerada anual, longa e de alta sincronia, com duração média de seis meses, no entanto, para algumas matrizes, a quantidade de frutos foi muito baixa, impossibilitando a coleta. Segundo TAPPER (1992) a frequência irregular de frutificação é considerada uma estratégia adaptativa para reduzir a predação de sementes.

A maturação por um longo período também pode estar associada à estratégia de perpetuação da espécie, que, quando dispersada por maior tempo, suas sementes recalcitrantes terão maiores chances de germinar. Outro fato importante é que a maturação de sementes de $\boldsymbol{C}$. canjerana no período chuvoso favorece a germinação, uma vez que suas sementes não suportam mais que poucos dias em solo seco.

A presença de frutos por um amplo período, apesar de proporcionar recurso para a fauna por maior tempo e, possivelmente, aumentar a chance para regeneração natural da espécie, dificulta a obtenção de um lote de sementes homogêneo em relação à maturação da semente. Consequentemente há necessidade de selecionar e marcar maior número de indivíduos para coleta, que, com base neste 
estudo, deve ser superior a 20, com a finalidade de permitir número de árvores matrizes com frutos em quantidades e nível de maturação semelhante no mesmo período.

No ano de 2008, quando se fez a comparação aos demais, houve $20 \%$ a menos das árvores matrizes marcadas frutificando; comparativamente com 2009, por exemplo, a média de pluviosidade, durante o desenvolvimento inicial dos frutos (setembro a novembro) foi mais elevada (311mm) em relação a 2008 (202mm) o que influenciou positivamente na frutificação.

$\mathrm{Na}$ maturação dos frutos, da mesma forma que na floração, observou-se discordância com CARVALHO (2003), o qual citou, para o Rio Grande do Sul, frutos maduros de junho a dezembro, enquanto BACKES \& IRGANG (2002) mencionaram ocorrer de junho a janeiro. As diferenças constatadas, seja por meio das observações ou pela comparação dos resultados numéricos obtidos para $\boldsymbol{C}$. canjerana, reforçam a necessidade de estudos regionais para avaliar o comportamento da espécie em diferentes condições, fornecendo informações quanto às restrições ambientais que interferem na frutificação. Da mesma forma, ao observar as árvores matrizes individualmente, constatou-se variação na maturação dos frutos, indicando a dificuldade de formação de lotes de sementes de canjerana, cujo baixo percentual germinativo, em muitos casos, pode ser atribuído à diferente intensidade de maturação dos frutos coletados.

As constatações descritas anteriormente podem estar associadas ao tempo necessário para a deiscência dos frutos pós-coleta, considerando que, em alguns indivíduos, $100 \%$ dos frutos estavam abertos após sete dias, enquanto outros atingiram percentuais inferiores. De forma comparativa, INOUE (1978), ao coletar frutos no Paraná e utilizando o mesmo método para extração das sementes, obtiveram a deiscência média de $84 \%$ após 12 dias, sendo tais diferenças, provavelmente, associadas ao grau de maturação das sementes entre matrizes.

A quantidade média de sementes de $\boldsymbol{C}$. canjerana por quilograma foi de 3.164 e o teor de água variou de 49 a $62 \%$, conforme a árvore matriz (Tabela 1), cujo número de sementes por quilograma foi expressivamente inferior, quando comparado ao trabalho de KUNIYOSHI (1983), indicando que as sementes coletadas neste trabalho são maiores.

Conforme Carvalho \& Nakagawa (2012), durante o desenvolvimento, as sementes crescem em tamanho, resultado da multiplicação e do desenvolvimento das células que constituem o eixo embrionário e o tecido de reserva. O tamanho, uma vez atingido o máximo, é mantido por certo tempo e, ao final do período de maturação, sofre redução mais ou menos acentuada, dependendo da espécie, o que corresponde à desidratação das sementes (CARVALHO \& NAKAGAWA, 2012). Assim, variações no tamanho de sementes entre indivíduos da mesma espécie, provavelmente, acontece em função da variabilidade genética entre matrizes e a influência ambiental durante seu desenvolvimento (FELIPPI et al., 2012).

$\mathrm{O}$ alto teor de água nas sementes pode estar relacionado à maturação e à característica

Tabela 1 - Avaliação do número de sementes por quilograma $(\mathrm{kg})$, coeficiente de variação (CV\%); teor de água (Ta), porcentagem (\%G) e índice de velocidade de germinação (IVG); emergência (\%E) e índice de velocidade de emergência (IVE).

\begin{tabular}{|c|c|c|c|c|c|c|}
\hline Árvore Matriz & $\begin{array}{c}\text { № de sementes por } \\
\mathrm{Kg} / \mathrm{CV} \%\end{array}$ & $\% \mathrm{Ta}$ & $\mathrm{G} \%$ & IVG & $\mathrm{E} \%$ & IVE \\
\hline 1 & $3.268 / 3,12$ & $55_{\mathrm{bac}}$ & $22_{c}$ & $0.30_{\mathrm{c}}$ & $19_{\mathrm{b}}$ & $0.30_{\mathrm{dc}}$ \\
\hline 2 & $3.395 / 2,41$ & $49_{\mathrm{c}}$ & $35_{\mathrm{bc}}$ & $0.62_{\mathrm{bc}}$ & $48_{\mathrm{a}}$ & $0.69_{\mathrm{ba}}$ \\
\hline 3 & $3.145 / 3,87$ & $54_{\mathrm{bc}}$ & $86_{a}$ & $2.39 \mathrm{a}$ & $31_{b}$ & $0.50_{\mathrm{bc}}$ \\
\hline 4 & $3.147 / 3,38$ & $58_{\mathrm{ba}}$ & $62_{\mathrm{ba}}$ & $1.57_{\mathrm{ba}}$ & $33_{\mathrm{b}}$ & $0.49_{\mathrm{bc}}$ \\
\hline 5 & $2.508 / 3,65$ & $55_{\text {bac }}$ & $69_{\mathrm{ba}}$ & $1.32_{\mathrm{b}}$ & $54_{a}$ & $0.74_{\mathrm{a}}$ \\
\hline 6 & $3.473 / 3,65$ & $61_{\mathrm{ba}}$ & $23_{\mathrm{c}}$ & $0.98_{\mathrm{bc}}$ & $22_{b}$ & $0.22_{\mathrm{d}}$ \\
\hline 7 & $3.210 / 3,29$ & $62_{\mathrm{a}}$ & $28_{\mathrm{c}}$ & $0.56_{\mathrm{c}}$ & $20_{\mathrm{b}}$ & $0.32_{\mathrm{dc}}$ \\
\hline $\begin{array}{l}\text { Pressuposições }{ }^{1} \mathrm{~W} \\
\text { Básicas } \chi^{2} \\
\mathrm{CV} \%\end{array}$ & & $\begin{array}{l}\mathrm{Pr}<\mathrm{W} 0.006 / \\
0.021 \\
\text { Alpha } \\
\begin{array}{l}0.0000 / 0.0002 \\
3.165\end{array}\end{array}$ & $\begin{array}{l}\operatorname{Pr}<W \\
0.006 / 0.008 \\
\text { Alpha } 0.094 \\
26.351\end{array}$ & $\begin{array}{l}\operatorname{Pr}<\mathrm{W} \\
0.002 / 0.278 \\
\text { Alpha } 0.001 / \\
0.000 \\
30.285\end{array}$ & $\begin{array}{l}\operatorname{Pr}<\mathrm{W} \\
0.044 / 0.0001 \\
\text { Alpha } 0.0002 / \\
4.865 \\
25.351\end{array}$ & $\begin{array}{l}\operatorname{Pr}<\mathrm{W} \\
0.002 / 0.007 \\
\text { Alpha } 0.001 / \\
0.000 \\
25.911\end{array}$ \\
\hline
\end{tabular}

${ }^{1} \mathrm{~W}$ : estatística do teste de Shapiro-Wilk e $\chi^{2}$ : estatística do teste de Bartlett; valores em negrito indicam normalidade dos resíduos e homogeneidade da variância, respectivamente, após transformação dos dados. As médias seguidas da mesma letra na coluna não diferem entre si pelo teste de Kruskal-Wallis e Dunn a 5\% de probabilidade de erro; as médias das tabelas correspondem aos dados originais. 
recalcitrante, porém não se pode relacioná-lo com a baixa porcentagem de germinação, apesar das sementes das matrizes 3 e 5, com teores intermediários, terem maiores porcentagens de germinação (Tabela 1). Essa germinação iniciou-se entre o $4^{\circ}$ e $16^{\circ}$ dia e não foi uniforme porque variou de 22 a $86 \%$ (Tabela 1), enquanto a emergência teve início no $20^{\circ}$ dia após a semeadura e variou de 19 a 54\%, podendo indicar que, para produção de mudas, é necessário maior quantidade de sementes, permitindo o semeio de mais de uma semente por recipiente.

Pelos dados da tabela 1, observa-se que as árvores matrizes com sementes maiores (menor número de sementes por quilograma) alcançaram porcentagens de germinação mais elevadas. Isso está de acordo com LEDO et al. (2002), os quais descreveram que o maior tamanho de sementes de canjerana esteja positivamente relacionado à quantidade de reservas, característica essa que aumentou a probabilidade de estabelecimento da plântula, em condições ambientais desfavoráveis.

A germinação de sementes de $\boldsymbol{C}$. canjerana mesmo sendo desuniforme foi rápida para algumas matrizes. Na matriz 3, houve $55 \%$ das sementes germinadas aos seis dias, enquanto, no mesmo período, a matriz 6 teve apenas $1 \%$ de germinação. Também houve diferença na emergência, sendo as matrizes 3 , 4 e 5 as que obtiveram porcentagens mais elevadas (31, 33 e $54 \%$, respectivamente), sobressaindo-se em relação às outras, principalmente quando comparadas a 1 e 6 (19 e $22 \%$, respectivamente).

Ao se observar germinação de sementes e emergência de plântulas das matrizes de canjerana, em relação a outros autores, verificou-se semelhança nas respostas, pois RIZZINI (1977), ao analisar lotes de sementes dessa espécie em condições de laboratório obteve germinação de 40 a $93 \%$, entre 3 e 10 dias, enquanto BACKES \& IRGANG (2002) observaram que a emergência ocorreu entre 13 e 73 dias após a semeadura.

As diferenças estatísticas observadas entre as sementes provenientes das matrizes de canjerana, com base nas variáveis utilizadas (Tabela 1), indicam que a baixa qualidade delas pode estar associada à variação da maturação fisiológica e, que aspectos como coloração dos frutos, quando utilizados isoladamente, são insuficientes para determinar o momento adequado da coleta de sementes dessa espécie.

Portanto, há necessidade de se buscar informações sobre o ponto de maturidade fisiológica das sementes, pois, diante dos resultados, o método tradicional de coleta (análise morfológica pelo tamanho e cor do fruto) é ineficiente, disponibilizando material com baixa qualidade fisiológica e lotes compostos por sementes em diferentes estádios de maturação. De qualquer forma, acredita-se que a implantação de áreas de produção de sementes de espécies florestais nativas, com o propósito de elevar a oferta de sementes de qualidade física e fisiológica, seja uma alternativa para produção de mudas florestais.

\section{CONCLUSÃO}

A floração de $\boldsymbol{C}$. canjerana é anual, persistindo de agosto a dezembro, sendo possível encontrar botões florais, concomitantemente com frutos em desenvolvimento e maduros, os quais iniciam a dispersão no mês de setembro, podendo ser coletados em amplo período (setembro a março), porém, frutos aparentemente maduros, em diferentes árvores matrizes, não garantem elevado poder germinativo às sementes de $\boldsymbol{C}$. canjerana.

\section{AGRADECIMENTOS}

A Universidade Federal de Santa Maria (UFSM) e a Coordenação de Aperfeiçoamento de Pessoal de Nível Superior (CAPES), pela bolsa de estudos concedida ao primeiro autor, durante seu curso de doutorado no Programa de Pós-graduação em Engenharia Florestal.

\section{REFERÊNCIAS}

BACKES, P.; IRGANG, B. Árvores do Sul: guia de identificação e interesse ecológico. Porto Alegre: Instituto Souza Cruz, 2002. 326p.

BENCKE, C.S.C.; MORELLATO, P.C. Estudo comparativo da fenologia de nove espécies arbóreas em três tipos de floresta atlântica no sudeste do Brasil. Revista Brasileira de Botânica, v.25, n.2, p.237-248, 2002. Disponível em: <http://www.scielo.br/ pdf/rbb/v25n2/11459>. Acesso em: 20 nov. 2009.

BRASIL. Ministério da Agricultura, Pecuária e Abastecimento Regras para análise de sementes. Ministério da Agricultura, Pecuária e Abastecimento. Secretaria de Defesa Agropecuária. Brasília: MAPA/ACS, 2009. 395p.

CALVIN, G.P.; PIÑA-RODRIGUES, F.C.M. Fenologia e produção de sementes de Euterpe edulis Mart. em trecho de floresta de altitude o município de Miguel Pereira - RJ. Revista da Universidade Rural, v.25, n.1, p.22-40, 2005. Disponível em: $\quad<$ http://www.scielo.br/scielo.php?script=sci_nlinks\&ref $=000083 \& \mathrm{pid}=\mathrm{S} 01006762201000060001200009 \& \operatorname{lng}=\mathrm{en}>$. Acesso em: 20 nov. 2009.

CARVALHO, P.E.R. Espécies arbóreas brasileiras. Colombo: EMBRAPA/CNPR, Brasília: EMBRAPA-SPI, 2003. V.1. 1039p. il.

CARVALHO, N.M.; NAKAGAWA, J. Sementes: ciência, tecnologia e produção. 5ed. Jaboticabal: FUNEP, 2012. 590p.

FELIPPI, M. et al. Fenologia, morfologia e análise de sementes de Apuleia leiocarpa (Vogel) J. F. Macbr. Ciência Florestal, 
v.22, n.3, p.477-491, 2012. Disponível em: <http://dx.doi. org/10.5902/198050986616>. Acesso em: 20 nov. 2014.

GUI-FERREIRA, A.; BORGHETTI, F. Germinação: do básico ao aplicado. Porto Alegre: Artmed, 2004. 323p. il.

INOUE, M.T. Indução à deiscência de frutos de Cabralea sp. Floresta, v.9, n.1, p.14-18, 1978. Disponível em: <http://ojs.c3sl. ufpr.br/ojs2/index.php/floresta/article/view/6210/4427>. Acesso em: 10 out. 2008

KANASHIRO, M. et al. Improving conservation values of managed forests: the Dendrogene Project in the Brazilian Amazon. Unasylva, v.53, n.1, p.25-33, 2002. Disponível em: <http://www.fao.org/// docrep/004/y3582e/y3582e06.htm>. Acesso em: 11 set. 2008.

KLEIN, R.M. Meliáceas. In: Reitz, R. (Ed.). Flora ilustrada catarinense I Parte. Itajaí: R. Reitz, 1984. 138p.

KUNIYOSHI, Y.S. Morfologia da semente e da germinação de 25 espécies arbóreas de uma floresta com araucária. 1983. 233f. Dissertação (Mestrado em Ciências Florestais) - Universidade Federal do Paraná, Curitiba, PR.

LEIGHT E.G. Tropical forest ecology. New York: Oxford University, 1999. 245p.

LEDO, A.S. et al. Efeito do tamanho da semente, do substrato e préembebição na germinação de sementes de pupunha. Revista Ciência Agronômica, v.33, n.1, p.29-32, 2002. Disponível em: <http://www. scielo.br/scielo.php?script $=$ sci nlinks\&ref $=000101 \&$ pid $=$ S0101312 2200900010003100013\&lng=en>. Acesso em: 20 nov. 2008.

MORELLATO, L.P.C. et al. Estudo comparativo da fenologia de espécies arbóreas de floresta de altitude e floresta mesófila semi-decídua na Serra do Japi, Jundiaí, São Paulo. Revista Brasileira de Botânica, v.12, n.2, p.85-98, 1989. Disponível em: $<$ http://www.scielo.br/scielo.php?pid=S0100$84042002000200012 \&$ script $=$ sci_arttext $>$ Acesso em: 5 set. 2008.

MORELLATO, L.P.C. As estações do ano na floresta. In: MORELLATO, L.P.C.; LEITÃO FILHO, H.F. Ecologia e preservação de uma floresta tropical urbana: reserva de Santa Genebra. Campinas: UNICAMP, 1995. 136p.
MOSCHETA, I.S. et al. Morfo-anatomia e aspectos da biologia floral de Cabralea canjerana (Vell) Mart. (Meliaceae). Acta Científica Venezolana, v.53, n.4, p.239-244, 2002. Disponível em: $<$ http:// www2.scielo.org.ve/scielo.php?script $=$ sci_arttext\&pid $=$ S000155042002000400002\&nrm=iso>. Acesso em: 5 set. 2008.

NEWSTROM, L.E. et al. A new classification for plant phenology based on flowering patterns in lowland tropical rain forest trees at La Selva, Costa Rica. Biotropica, v.26, n.2, p.141-159, 1994. Disponível em: <http://www.jstor.org/discover/10.2307/2 388804 ? uid $=3737664$ \&uid $=2$ \&uid $=4 \&$ sid $=21102037920491>$. Acesso em: 20 nov. 2008.

PENNINGTON, T.D. A monograph of the neotropical Meliaceae. In: PENNINGTON, T.D. et al. Flora neotropica Monograph 28: Meliaceae. New York: The New York Botanical Garden, 1981. p.1-449.

REITZ, R. et al. Projeto madeira do Rio Grande do Sul. Porto Alegre: Secretaria de Estado da Agricultura e Abastecimento, 1988. 525p.

RIO GRANDE DO SUL. Governo do Estado. Secretaria Estadual do Meio Ambiente. Relatório final do inventário florestal contínuo do Rio Grande do Sul. Porto Alegre: SEMA/UFSM, 2001. V.1,2. 706p.

RIZZINI, C.T. Tratado da fitogeografia do Brasil: Aspectos ecológicos, sociológicos e florísticos. Rio de Janeiro: Âmbito Cultural. 2ed. 1977. 747p.

SANTANA, D.G.; RANAL, M.A. Análise da germinação: um enfoque estatístico. Brasília: Universidade de Brasília, 2004. 248p.

SILVA, L.D.; HIGA, A.R. Planejamento e implantação de pomares de sementes de espécies florestais nativas. In: HIGA, A.R.; SILVA, L.D. Pomar de sementes de espécies florestais nativas. Curitiba: FUPEF, 2006. p.93-138.

TAPPER, P.G. Irregular fruiting in Fraxinus excelsior. Journal of Vegetation Science, v.3, n.1, p.41-46, 1992. Disponível em: $<$ http://onlinelibrary.wiley.com/doi/10.2307/3235996/pdf $>$. Acesso em: 10 dez. 2014.

ZAR, J.H. Biostatistical analysis. 5.ed. New Jersey: PrenticeHall, 2009. 960p. 